\title{
Effect of well bore mixing volume on non-axisymmetrical transport in a convergent radial tracer test
}

\author{
Jui-Sheng Chen ${ }^{\mathrm{a}}$, Chen-Wuing Liu ${ }^{\mathrm{b}, *}$, Chia-Shyun Chen ${ }^{\mathrm{c}}$, Chung-Min Liao ${ }^{\mathrm{b}}$ \\ ${ }^{a}$ Department of Environmental Engineering and Sanitation, Fooyin Institute of Technology, Kaohsiung, Taiwan, ROC \\ ${ }^{\mathrm{b}}$ Department of Bioenvironmental Systems Engineering, National Taiwan University, 1, Roosevelt Road, Sec. 4, Taipei 10617, Taiwan, ROC \\ ${ }^{\mathrm{c}}$ Institute of Applied Geology, National Central University, Taoyuan, Taiwan, ROC
}

Received 8 October 2001; accepted 17 February 2003

\begin{abstract}
When tracers are introduced into an injection borehole, the resulting non-axisymmetrical plume undergoes transverse dispersion during transport to the extraction well in a radially convergent tracer test. The transverse dispersivity governs the shape of the breakthrough curves observed at a point between the extraction and injection wells. Accordingly, concentrations monitored at the extraction and observation wells can be used to simultaneously determine the longitudinal and transverse dispersivities. This study presents a two-dimensional Laplace transform finite-difference (LTFD) model to examine the effect of the finite mixing volume of an injection well bore on non-axisymmetrical transport during a radially convergent tracer test. The developed model was compared to the analytical solution to evaluate the accuracy of the model. Comparative results suggest that the LTFD model with an upstream weighting technique incorporating an extra fine mesh, can be used to effectively and accurately solve the radial advection-dispersion equation with large Peclet numbers. Two tracer tests with distances 5 and $25 \mathrm{~m}$, were considered to investigate the effect of inter-well distance on the role of injection well bore storage. Simulation results reveal that injection well bore mixing volume significantly decreases the peak concentration and spreads out the breakthrough curve obtained in the tracer test with a $5 \mathrm{~m}$ inter-well distance. When the inter-well distance increases to $25 \mathrm{~m}$, the injection well bore mixing volume weakly affects the tracer transport.
\end{abstract}

(C) 2003 Elsevier Science B.V. All rights reserved.

Keywords: Transverse dispersion; Well bore mixing volume; Laplace transform finite-difference method; Non-axisymmetrical transport

\section{Introduction}

Tracer tests attempt to determine solute transport parameters, such as aquifer porosity, the dispersion tensor and hydrogeological properties. A radially convergent tracer test facilitates the recovery of

\footnotetext{
* Corresponding author. Tel.: + 886-2-23626480; fax: + 886-223639557.

E-mail address: lcw@gwater.agec.ntu.edu.tw (C.-W. Liu).
}

injected mass, reduces the effect of apparent dispersion due to a flow field, and minimizes the influence of a natural hydraulic gradient. Thus, radially convergent tracer tests are particularly useful when transport characteristics, rather than hydraulic properties, are to be determined. These studies and experiments in this area are commonly limited to the analysis of breakthrough curves in the extraction well, although new sampling technologies are available for 
measuring concentration in the field (Mackay et al., 1994). The models' complexity is such that the analysis is often reduced to adjusting one-dimensional solutions (Thorbjarnson and Mackay, 1994), rather than involving the more accurate two-dimensional models.

Predictive and interpretive models of radially convergent tracer tests have been developed as axisymmetrical models (Sauty, 1980; Guvanasen and Guvanasen, 1987; Moench, 1989, 1991, 1995; Welty and Gelhar, 1994; Wang and Crampon, 1995; Chen et al., 1996; Becker and Charbeneau, 2000). Axisymmetrical models typically consider only longitudinal dispersivity. In a related work, Guvanasen and Guvanasen (1987) first took the transverse dispersion into consideration and derived an approximate twodimensional, semi-analytical solution in streamline and equipotential coordinates to describe the nonaxisymmetrical tracer transport. Their solution, however, is appropriate for the generation of breakthrough curves only. Guvanasen and Guvanasen (1987) observed the discrepancy between their approximate solution and the verified finite element solution for small Peclet numbers. Given the importance of solving this non-axisymmetrical problem, Chen et al. (1999) presented a two-dimensional mathematical model in cylindrical coordinates to illustrate the non-axisymmetrical tracer transport in a radially convergent tracer test. They also proposed a curvefitting method that involved a theoretical breakthrough curve at an intermediate point, to evaluate transverse dispersivity, which could not be determined by a one-dimensional model. The method does not, however, allow mixing in either the extraction or the injection well bores.

Moench (1989) considered the effect of both extraction and injection well mixing volumes in radially convergent tracer tests, suggesting that both extraction and injection well bore mixing volumes can markedly affect systems with low porosity. In particular, both extraction and injection well bore mixing volumes can cause significant spreading of the breakthrough curves and can shift the arrival time of the peak concentration in such systems. Moreover, from the definitions of extraction and injection well bore mixing factors (Moench, 1989), both effects are almost identical if the diameters of the extraction and injection wells are equal.
Zlotnik and Logan (1996) pointed out that Moench (1989) improperly formulated the boundary conditions at the injection well. Zlotnik and Logan (1996) derived an improved boundary condition based on a detailed analysis of flow and advective transport in the injection well, demonstrating that the improper use of the model derived by Moench (1989) could produce incorrect breakthrough curves for the extraction well under conditions that involve a significant mixing factor in the injection well. A numerical example indicated an error of up to $70 \%$ in the peak concentrations of a breakthrough curve and of $10 \%$ in the peak arrival time for a Peclet number of 100 (Zlotnik and Logan, 1996).

This work investigates the effect of injection well bore mixing volume on non-axisymmetrical transport in a radially convergent tracer test, using the formulations of Zlotnik and Logan (1996). A Laplace transform finite-difference (LFTD) model is developed to evaluate the effect of well bore mixing volume on the movement of a tracer. The result represents an improvement in the accuracy of the determined solute transport parameters, including aquifer porosity, and longitudinal and transverse dispersivities.

\section{Mathematical model}

The tracer test in a radially convergent flow field is considered. The flow field is generated by a fully penetrating well of radius $r_{\mathrm{W}}$ located on the vertical axis at $r=0, \theta=0$, and fluid pumped at a constant volume rate of $Q$ from a homogeneous and isotropic aquifer of infinite horizontal extent (Fig. 1). The seepage velocity $V$ caused by extraction is given by,

$V=-\frac{A}{r}$

where $A=Q / 2 \pi b \phi$, and $b$ and $\phi$ represent the aquifer thickness and effective porosity, respectively. When a field test is initiated, once water levels are stabilized, a tracer is introduced into the injection borehole with a radius $r_{\mathrm{I}}$ and a center point $\left(r_{\mathrm{L}}, \pi\right)$ from which it flows out of the injection borehole (Fig. 1). The concentration distribution $C(r, \theta, t)$ is not axisymmetrical. The configuration in Fig. 1 is symmetrical around the line that passes through the extraction and injection wells. Thus, only one half-plane is considered. 
A
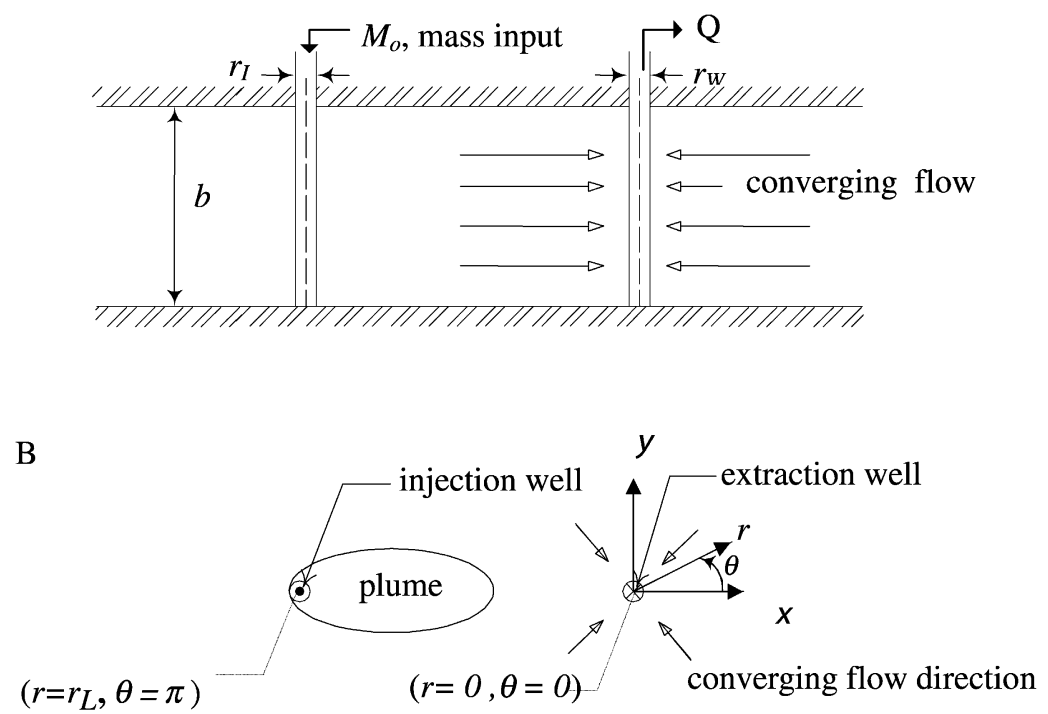

Fig. 1. Schematic diagram of a convergent radial tracer test after Chen et al. (1999): (A) cross section; (B) plane view.

Mechanical dispersion is assumed to follow Fick's law in that the longitudinal and transverse dispersion coefficients are proportional to the velocity. The effects of molecular diffusion are assumed to be much smaller than those of mechanical dispersion.

The tracer transport equation in cylindrical coordinates is,

$\frac{a_{\mathrm{L}} A}{r} \frac{\partial^{2} C}{\partial^{2} r}+\frac{A}{r} \frac{\partial C}{\partial r}+\frac{a_{\mathrm{T}} A}{r^{3}} \frac{\partial^{2} C}{\partial^{2} \theta}=R \frac{\partial C}{\partial t}$

where $a_{\mathrm{L}}$ and $a_{\mathrm{T}}$ denote the longitudinal and transverse dispersivities, respectively, and $R$ is a retardation factor.

The aquifer's initial tracer concentration is assumed to be zero before the test is started

$C(r, \theta, 0)=0, \quad r_{\mathrm{W}} \leq r \leq r_{\mathrm{L}}$

A mass balance at the well screen, used to ensure perfect mixing, is considered to account for the influence of mixing in the extraction well. The outlet boundary condition, which describes the solute transport between the extraction well and aquifer is,

$\pi r_{\mathrm{W}}^{2} h_{\mathrm{W}} \frac{\partial C_{\mathrm{W}}(t)}{\partial t}=2 \pi r_{\mathrm{W}} \phi b a_{\mathrm{L}}\left|\frac{A}{r_{\mathrm{W}}}\right| \frac{\partial C(r, \theta, t)}{\partial r}$ at

$r=r_{\mathrm{W}}$ where $h_{\mathrm{W}}$ is the mixing length of the extraction well, and $C_{\mathrm{W}}(t)$ denotes the tracer concentration in the extraction well (Hodgkinson and Lever, 1983; Moench, 1989).

The initial condition applied to Eq. (4) implies that the well bore contains no contaminant before pumping begins, and is given by,

$C_{\mathrm{W}}(0)=0$

If the volume of water in the well bore can be neglected, then Eq. (4) becomes the commonly used condition $\partial C / \partial r=0$ at the extraction well $(r=$ $\left.r_{\mathrm{W}}\right)$.

Zlotnik and Logan (1996) considered flow and transport in a ring-shaped domain centered at the extraction well, bounded by circles of radii $r=r_{\mathrm{W}}$ and $r_{*}=r_{\mathrm{L}}-l\left(l \ll r_{\mathrm{L}}\right)$, in deriving the boundary condition at the injection well. The physical assumption is that advective transport dominates dispersive transport at a small distance $l \approx 5 r_{\mathrm{I}}$ downstream in the discharge zone of the injection well.

Therefore the concentration distribution $C(r, \theta, t)$ at a distance $r=r_{*}$ from the extraction well obeys the 
following boundary condition,

$a_{\mathrm{L}} \frac{A}{r} \frac{\partial C(r, \theta, t)}{\partial r}+\frac{A}{r} C(r, \theta, t)$

$$
=\left\{\begin{array}{ll}
\frac{A}{r} C_{\mathrm{I}}(t) & \pi-\delta<\theta<\pi \\
0 & 0<\theta<\pi-\delta
\end{array} r=r_{*} \approx r_{\mathrm{L}}\right.
$$

where $C_{\mathrm{I}}(t)$ is the concentration generated in the injection well and transported downstream through the narrow and short (a few well diameters) discharge zone by advection. This small zone with advection-dominated flow has an aperture angle of $2 \delta$. The aperture angle of this narrow zone at the distance $r_{*} \approx r_{\mathrm{L}}-l$ from the center of extraction well is,

$2 \delta=\frac{2 \alpha r_{\mathrm{I}}}{r_{\mathrm{L}}}$

where $\alpha$ is a factor that defines the distortion of distances between the two most widely separated stream lines that enter (or leave) the injection well. This parameter also depends on the skin effect for an injection well (Zlotnik and Logan, 1996; Eq. (3)). $\alpha=2$ for uniform isotropic aquifer with a well without a skin. For skins with high conductivity, $2 \leq \alpha \leq 4$, whereas for skins with low conductivity, $0<\alpha \leq 2$ (Drost et al., 1968).

The effluent concentration from the injection well in an ambient horizontal flow is yet to be determined. The effluent concentration from the well with an initial dissolved tracer mass, $M_{0}$, satisfies a mass balance equation for the tracer in the borehole:

$-2 \alpha r_{\mathrm{I}} \phi b\left|V\left(r_{\mathrm{L}}\right)\right| C_{\mathrm{I}}=\pi r_{\mathrm{I}}^{2} h_{\mathrm{I}} \frac{\mathrm{d} C_{\mathrm{I}}}{\mathrm{d} t}$

$C_{\mathrm{I}}(0)=\frac{M_{0}}{\pi r_{\mathrm{I}}^{2} h_{\mathrm{I}}}=C_{0}$

where $h_{\mathrm{I}}$ is the mixing length of the injection well (Zlotnik and Logan, 1996; Eq. (6)).

After integration, the known effluent concentration $C_{\mathrm{I}}(t)$ can be substituted into the boundary condition, Eq. (6). The physics of the problem stipulate that $C$ is a single-valued function in $r$ and $\theta$ coordinates. Moreover, $C$ is a continuous and symmetrical function across $\theta=0$ and $\theta=\pi$. Thus, the boundary conditions in the transverse direction are as follows

$$
\begin{aligned}
& \frac{\partial C(r, 0, t)}{\partial \theta}=0 \\
& \frac{\partial C(r, \pi, t)}{\partial \theta}=0
\end{aligned}
$$

The definitions of the dimensionless variables are similar to those used by Chen et al. (1999). Following Moench (1989), substituting the definitions given in Table 1 into Eq. (2) yields the dimensionless transport equation in the following form

$$
\begin{gathered}
\frac{1}{P e} \frac{1}{r_{\mathrm{D}}} \frac{\partial^{2} C}{\partial r_{\mathrm{D}}^{2}}+\frac{1}{r_{\mathrm{D}}} \frac{\partial C}{\partial r_{\mathrm{D}}}+\frac{1}{P e} \frac{a_{\mathrm{D}}}{r_{\mathrm{D}}^{3}} \frac{\partial^{2} C}{\partial \theta^{2}} \\
=\frac{2 R}{1-r_{\mathrm{WD}}^{2}} \frac{\partial C}{\partial t_{\mathrm{D}}}
\end{gathered}
$$

Table 1

Dimensionless parameters used in the mathematical model (after Moench, 1989; Zlotnik and Logan, 1996)

\begin{tabular}{ll}
\hline Dimensionless quantity & Expression \\
\hline Time & $t_{\mathrm{D}}=\frac{Q t}{\pi h \phi\left(r_{\mathrm{L}}^{2}-r_{\mathrm{W}}^{2}\right)}$ \\
& $r_{\mathrm{D}}=\frac{r}{r_{\mathrm{L}}}$ \\
Distance & $r_{\mathrm{WD}}=\frac{r_{\mathrm{W}}}{r_{\mathrm{L}}}$ \\
Extraction well radius & $r_{\mathrm{ID}}=\frac{r_{\mathrm{I}}}{r_{\mathrm{L}}}$ \\
Injection well radius & $P e=\frac{r_{\mathrm{L}}}{a_{\mathrm{L}}}$ \\
Injection well mixing factor & $\mu_{\mathrm{I}}=\frac{r_{\mathrm{I}} r_{\mathrm{L}} h_{\mathrm{I}}}{\phi h\left(r_{\mathrm{L}}^{2}-r_{\mathrm{W}}^{2}\right)}$ \\
Extraction well mixing factor & $a_{\mathrm{D}}=\frac{a_{\mathrm{T}}}{a_{\mathrm{L}}}$ \\
Dispersivity ratio & $\mu_{\mathrm{W}}=\frac{r_{\mathrm{W}}^{2} h_{\mathrm{W}}}{\phi h\left(r_{\mathrm{L}}^{2}-r_{\mathrm{W}}^{2}\right)}$ \\
&
\end{tabular}


Consequently, the initial conditions and boundary conditions become,

$$
\begin{aligned}
& C\left(r_{\mathrm{D}}, \theta, 0\right)=0 \\
& \mu_{\mathrm{W}} \frac{\partial C_{\mathrm{W}}\left(t_{\mathrm{D}}\right)}{\partial t_{\mathrm{D}}}=\frac{1}{P e} \frac{\partial C\left(r_{\mathrm{D}}, \theta, t_{\mathrm{D}}\right)}{\partial r_{\mathrm{D}}} \text { at } r=r_{\mathrm{W}} \\
& C_{\mathrm{W}}(0)=0 \\
& \frac{1}{P e} \frac{\partial C\left(r_{\mathrm{D}}, \theta, t_{\mathrm{D}}\right)}{\partial r_{\mathrm{D}}}+C\left(r_{\mathrm{D}}, \theta, t_{\mathrm{D}}\right) \\
& =\left\{\begin{array}{l}
C_{\mathrm{I}}\left(t_{\mathrm{D}}\right) \\
0
\end{array} \quad \pi-\delta<\theta<\pi\right. \\
& -C_{\mathrm{I}}=\mu_{\mathrm{I}} \frac{\mathrm{d} C_{\mathrm{I}}}{\mathrm{d} t_{\mathrm{D}}} \\
& C_{\mathrm{I}}(0)=C_{0} \\
& \frac{\partial C\left(r_{\mathrm{D}}, 0, t_{\mathrm{D}}\right)}{\partial \theta}=0 \\
& \frac{\partial C\left(r_{\mathrm{D}}, \pi, t_{\mathrm{D}}\right)}{\partial \theta}=0
\end{aligned}
$$

This study adopts the LTFD method to solve the initial boundary value problem specified by Eqs. (12)-(20). Appendix A provides a detailed derivation of the LTFD method.

\section{Model verification}

\subsection{Verification of the model accuracy}

The axisymmetrical Laplace-domain analytical solution for a convergent tracer test of the Moench (1989, 1991) solution is used to test the accuracy of the developed LTFD model. Table 2 summarizes the simulation conditions and transport parameters for verification of the two practical tracer tests. Notably, the LTFD model determines the solution analytically in the $\theta$ coordinate and numerically in the radial coordinate. A uniform grid mesh was used to discretize the radial distance in this LTFD model. Numerical procedures based on the finiteelement or finite-difference methods performed well for dispersion-dominated transport (small Peclet number), but suffered from excessive artificial oscillation and numerical dispersion when applied to advection-dominated transport (large Peclet
Table 2

Numerical values used in the model verification for two practical tracer tests

\begin{tabular}{lll}
\hline Parameter & Test 1 & Test 2 \\
\hline Pumping rate, $Q\left(\mathrm{~m}^{3} / \mathrm{min}\right)$ & 2 & 2 \\
Aquifer thickness, $h(\mathrm{~m})$ & 10 & 10 \\
Effective porosity, $\phi($ dimensionless $)$ & 0.2 & 0.2 \\
Radius of extraction well, $r_{\mathrm{W}}(\mathrm{m})$ & 0.1 & 0.1 \\
Extraction well mixing length, $h_{\mathrm{W}}(\mathrm{m})$ & 10 & 10 \\
Radius of injection well, $r_{\mathrm{I}}(\mathrm{m})$ & 0.1 & 0.1 \\
Injection well mixing length, $h_{\mathrm{I}}(\mathrm{m})$ & 10 & 10 \\
Distance to the injection well, $r_{\mathrm{L}}$ & 5 & 25 \\
Injected mass, $M(\mathrm{~kg})$ & 10 & 40 \\
Longitudinal dispersivity, $a_{\mathrm{L}}(\mathrm{m})$ & $5,0.5,0.05$ & $25,2.5,0.25$ \\
Peclet number, $P e($ dimensionless $)$ & $1,10,100$ & $1,10,100$ \\
Transverse dispersivity, $a_{\mathrm{T}}(\mathrm{m})$ & $1,0.1,0.01$ & $5,0.5,0.05$ \\
Dispersivity ratio, $a_{\mathrm{D}}($ dimensionless $)$ & 0.2 & 0.2 \\
\hline
\end{tabular}

number). The problem of artificial oscillation can be solved by upstream weighting. Upstream weighting, however, tends to aggravate the numerical dispersion problem. The study used upstream weighting to prevent the artificial oscillation, and employs an extra fine grid mesh to eliminate numerical dispersion. The grid numbers, $N_{\text {grid }}$, required to eliminate numerical dispersion are considered here. Fig. 2 compares the breakthrough curves of Moench's analytical solution and those of the LTFD solution for various grid numbers. Two solutions agree closely for a small Peclet number of $P e=1$ (Fig. 2). The LTFD model with a coarse grid mesh $\left(N_{\text {grid }}=100\right)$ yields numerical results with noticeable numerical dispersion for $P e=10$. The dispersion becomes significant for $P e=100$. The LTFD solutions of the fine grid mesh $\left(N_{\text {grid }}=\right.$ 1000) agree well with analytical solutions for $P e=$ 1 and 10, yet the error from the numerical dispersion becomes noticeable at $P e=100$. By employing the extra fine grid mesh $\left(N_{\text {grid }}=\right.$ 10,000), the LTFD solution agrees closely with the analytical solution for all ranges of Peclet numbers. Increasing the grid numbers reduces and eventually eliminates the numerical dispersion of the difference between the concentration in the analytical solution and that in the LTFD solution. Although the method is computationally intensive, requiring, for example, a combination of numerical 


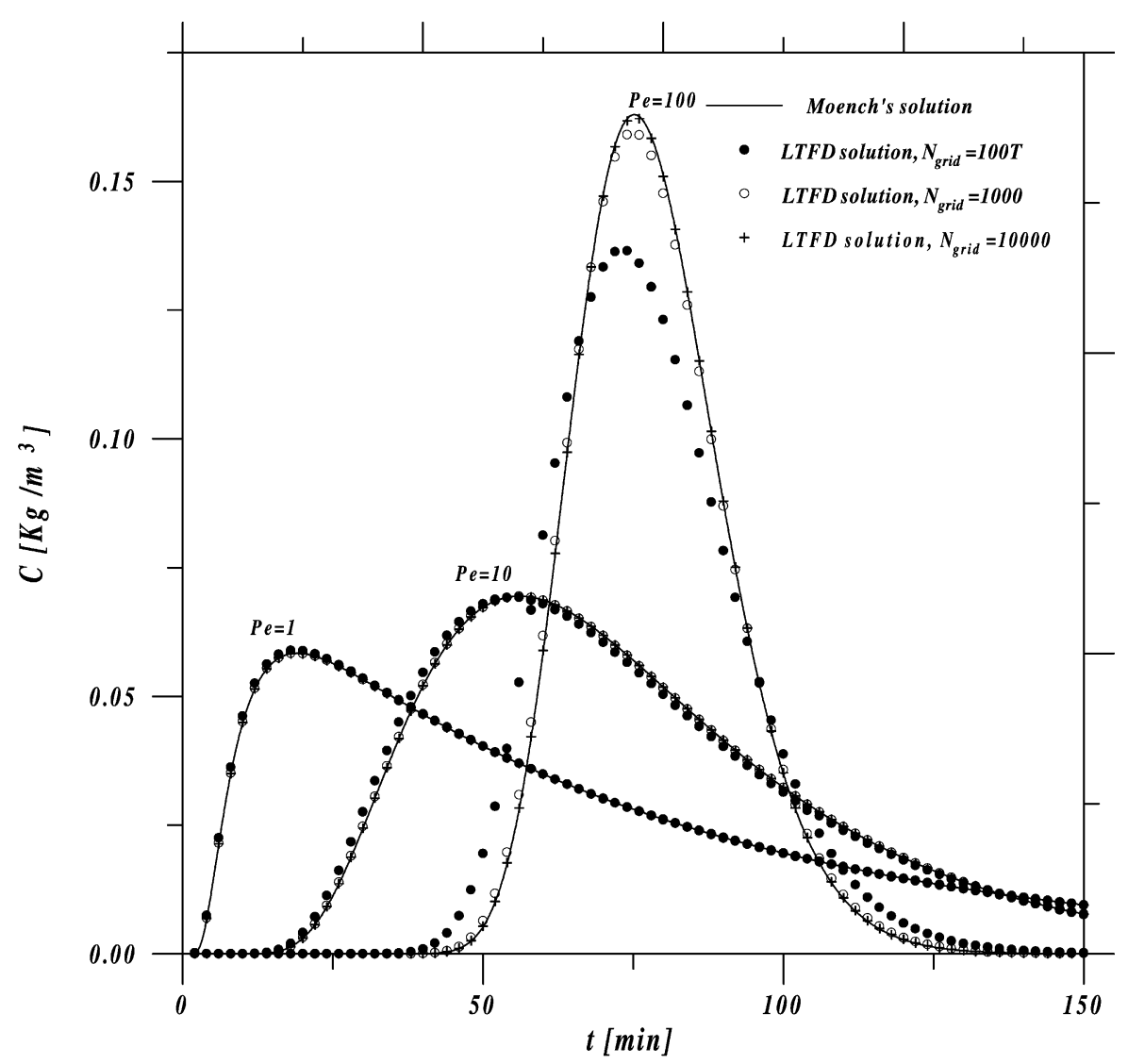

Fig. 2. Comparison of the developed LTFD model with different grid numbers and Moench's solution for $P e=1,10$, and 100 .

Laplace transform inversion, finite Fourier cosine transform inversion and finite-difference techniques, the overall CPU (central process unit) time used in the above three cases is less than $30 \mathrm{~min}$ on a Pentium III ${ }^{\circledR}$ computer.

Comparing the analytical solutions with the LTFD numerical solutions reveals that the LTFD model with an upstream weighting technique and an extra fine grid mesh can effectively and accurately solve the radial advection-dispersion equation with a large Peclet number.

\subsection{Mathematical behavior of the finite Fourier cosine inversion}

The finite Fourier cosine inversion in Eq. (A13) is given in the form of an infinite series. Infinite series can be straightforwardly evaluated. Yet, the fact that sufficient terms of the series must be summed to yield an accurate result needs to be considered. The dependence of the required number of terms on Peclet number provides an insight into the convergence of the finite Fourier cosine inversion. Table 3 presents the required number of series terms for $r_{\mathrm{D}}=5$ and for various Peclet numbers and dimensionless dispersivity ratios at a tolerance of $10^{-6}$. Give a fixed tolerance error, the number of terms to be summed increases with Peclet number and decreases as the dimensionless dispersivity ratio $a_{\mathrm{D}}$ increases. The required numbers of series terms in the finite Fourier cosine inversion are 8, 24, and 48 for $P e=1,10$, and 100, respectively. For the same fixed tolerance and $P e=10$, the required numbers of series terms in the finite Fourier cosine 
Table 3

The dependence of the number of the series terms for convergence of finite Fourier cosine inversion

\begin{tabular}{llll}
\hline Number of terms & $P e=1, a_{\mathrm{D}}=0.2$ & $P e=10, a_{\mathrm{D}}=0.2$ & $P e=10, a_{\mathrm{D}}=0.4$ \\
\hline$n$ & 8 & 24 & 16 \\
\hline
\end{tabular}

inversion are 24 and 16 for $a_{\mathrm{D}}=0.2$ and 0.4 , respectively.

\section{Effect of well bore mixing volume}

The developed LTFD model is applied to illustrate the effect of injection well bore mixing volumes on non-axisymmetrical contaminant transport in a radially convergent tracer test. Grid number 10,000 was used to discretize the mesh radially. Fig. 3 compares the concentration contours in Test 1 of the hypothetical tracer tests with and without the injection well bore mixing volume for 30, 60 and $90 \mathrm{~min}$, with a Peclet number of 100 and an inter-well distance of $5 \mathrm{~m}$. The solid and dashed lines in Fig. 3 represent the concentration contours of a tracer with and without the injection well bore mixing volume, respectively. Fig. 3 reveals that the advancement of the solute plume in a tracer test with a finite mixing volume of the injection well bore is slower than that obtained with an infinitesimal volume.

Data from the tracer test are commonly analyzed by matching the observed and theoretical breakthrough curves. Chen et al. (1999) pointed out that the longitudinal and transverse dispersivities can be simultaneously determined from breakthrough curves at the extraction well and an observation well in field tracer tests. They considered the influence of injection well mixing volume on breakthrough curves at both wells. Fig. 4 compares the breakthrough curves at the extraction well and at an observation point in Test 1 of the hypothetical tracer tests $(r=1, \theta=\pi)$, depicting the effects with and without injection well bore mixing. Apparently, injection well bore mixing significantly spreads the breakthrough curves and shifts the arrival time of the peak concentration (Zlotnik and Logan, 1996). A shorter distance from the observation well to the injection point causes greater spreading and a greater shift in the peaks of the breakthrough curves.

Fig. 5 is reconstructed from Fig. 4 by normalizing the concentration with respect to peak concentration $\left(C_{\mathrm{D}}=C / C_{\text {peak }}\right)$ at the extraction well, and by employing dimensionless time $\left(t_{\mathrm{D}}\right)$, which had been used to generate the type curves in determining longitudinal dispersivity. In general, the field breakthrough curves are first compared to the type curves at the extraction well to yield longitudinal dispersivity. The type curve for $P e=1$ with finite mixing volume in an injection well bore is chosen as the set of non-dimensionalized hypothetical field data to demonstrate how the finite mixing volume in the injection well bore affects the determination of longitudinal dispersivity for a radially convergent tracer test. The data are fitted to the type curves at the extraction well in a tracer test, neglecting the finite mixing volume of the injection well bore. Fig. 6 presents the hypothetical data and a match using type curves at the extraction well for a tracer test without injection well bore mixing. The estimated $P e$ is 1.61 rather than $P e=1$. Consequently, the estimate of dispersivity differs by a factor of $1 / 1.61 \approx 0.62$ if well bore mixing is not adequately considered.

In view of the definition of the injection well mixing factor, $\mu_{\mathrm{I}}$ (Table 1), which indicates the distance between two wells $\left(r_{\mathrm{L}}\right)$, significantly influences $\mu_{I}$. Therefore, the possibility of an injection well bore mixing effect of non-axisymmetrical transport on the inter-well distance, is examined. Fig. 7(a) and (b) shows how injection well bore mixing volume affects breakthrough curves at the extraction well and at the observation point at $r=5 \mathrm{~m}$, and $\theta=\pi$ in Test 2 . The effect of injection well bore mixing volume is insignificant 

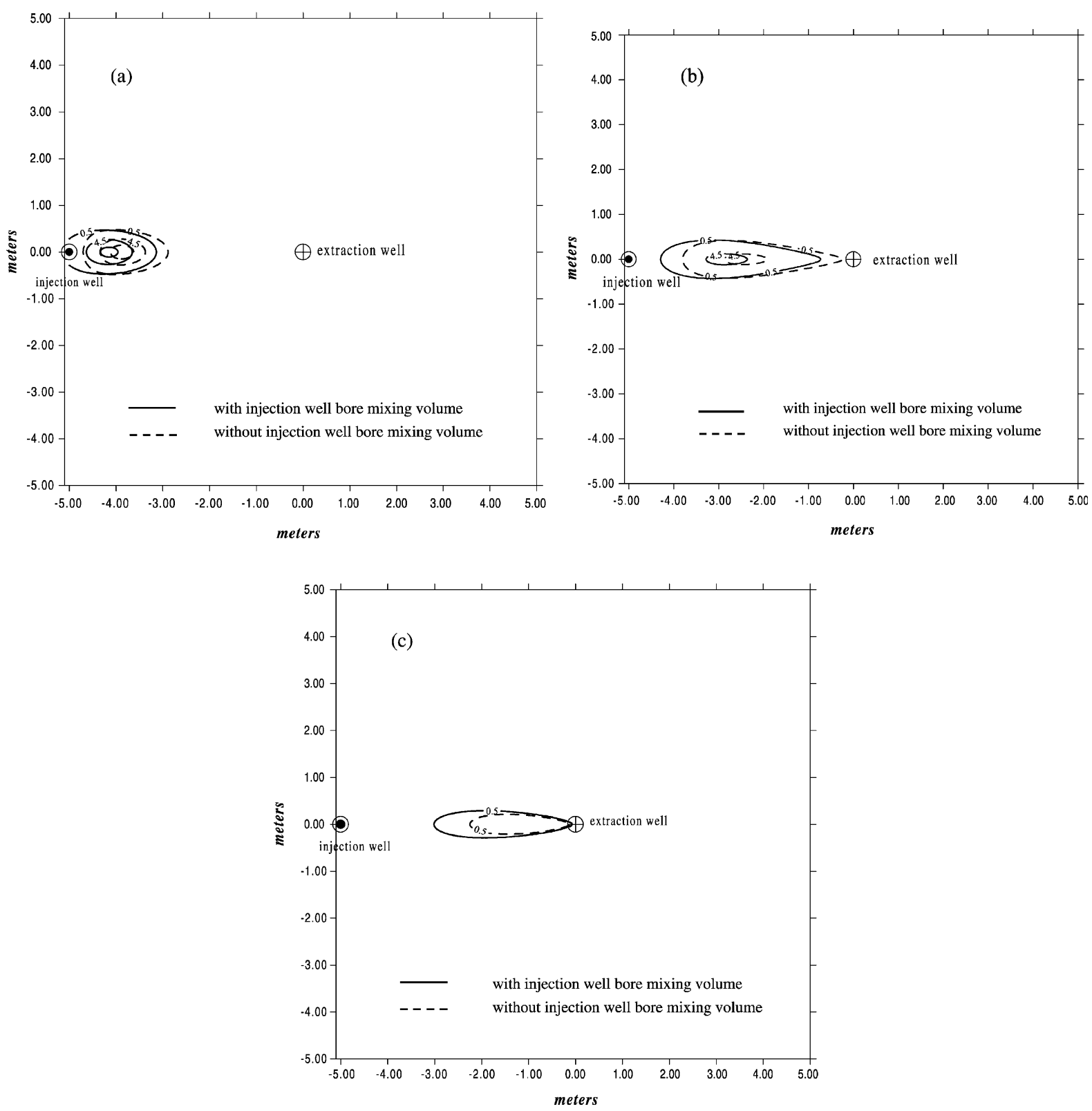

Fig. 3. Concentration contours of a hypothetical tracer test with and without injection well bore mixing volume: (a) $t=30$; (b) $t=60$; (c) $t=90 \mathrm{~min}$.

at the extraction well but is noticeable at the observation point at $r=5 \mathrm{~m}$, and $\theta=\pi$ in the scale tracer test with an inter-well distance of $25 \mathrm{~m}$, demonstrating that the longdistance tracer transport gradually reduces the retardation due to the injection well bore mixing volume.

Our study demonstrates that the finite mixing volume of an injection well bore significantly affects the results of the tracer test with a small inter-well 

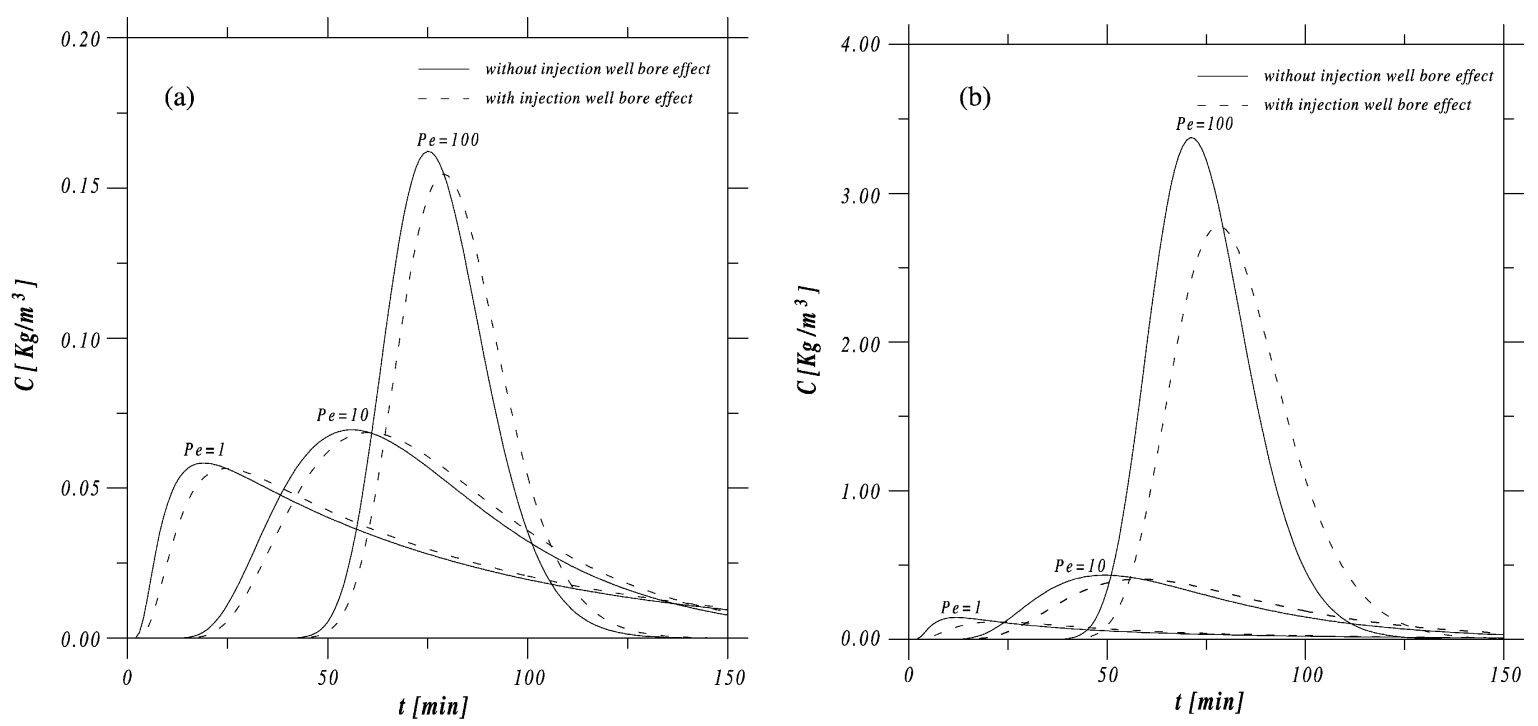

Fig. 4. Comparison of dimensional breakthrough curves with and without injection well bore effect: (a) extraction well for Test 1 tracer test with $r_{\mathrm{L}}=5 \mathrm{~m}$. (b) observation point $(r=1 \mathrm{~m}, \theta=\pi)$ for Test 1 tracer test with $r_{\mathrm{L}}=5 \mathrm{~m}$.

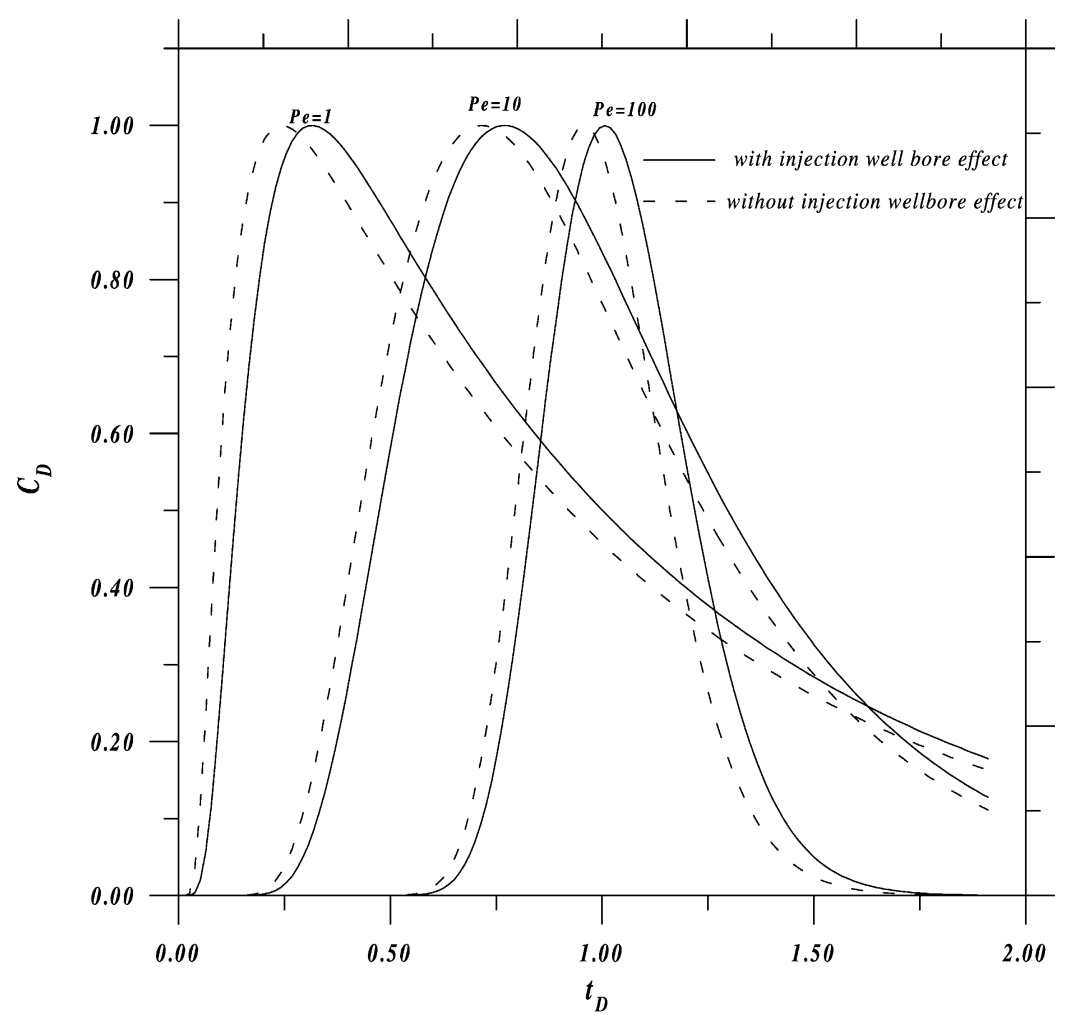

Fig. 5. Comparison of dimensionless breakthrough curves at extraction well with and without injection well bore effect with concentration normalized with respect to peak concentration and with the abscissa expressed as dimensionless time for Test 1 tracer test with $r_{\mathrm{L}}=5 \mathrm{~m}$. 


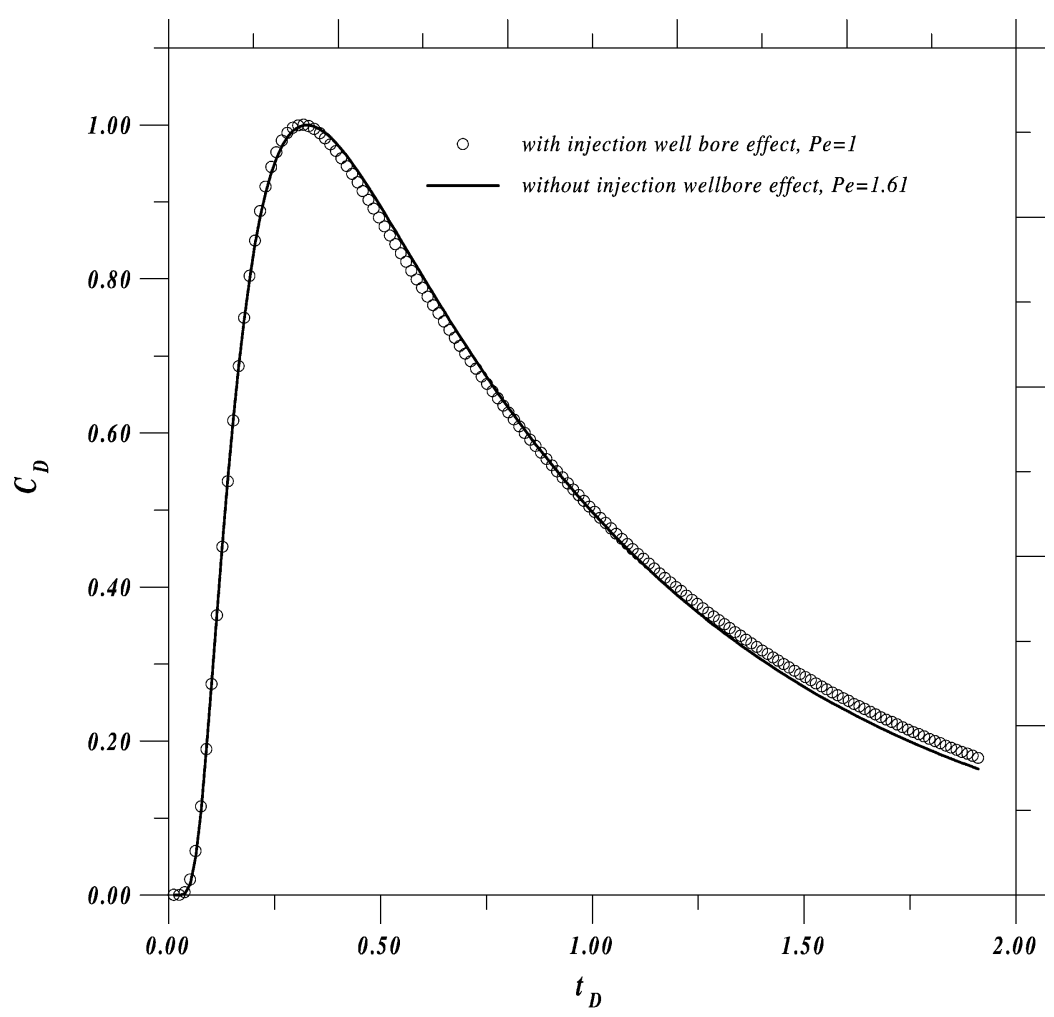

Fig. 6. Hypothetical field data (with injection well bore mixing volume) matched with the type curves of a tracer test without injection well bore mixing volume.
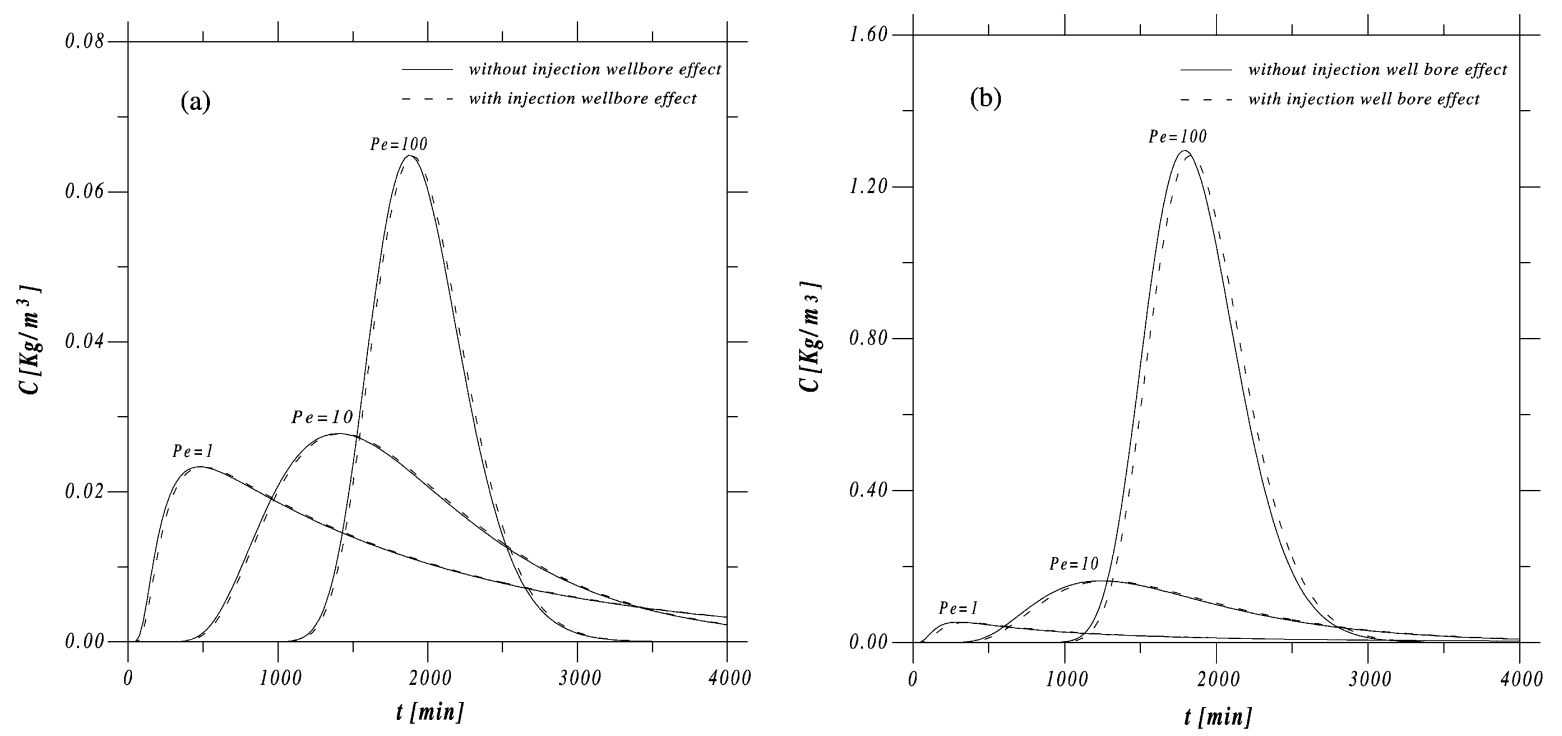

Fig. 7. (a) Comparison of dimensional breakthrough curves with and without injection well bore effect: (a) extraction well in a tracer test with $r_{\mathrm{L}}=25 \mathrm{~m}$; (b) observation point $(r=5 \mathrm{~m}, \theta=\pi)$ in a tracer test with $r_{\mathrm{L}}=25 \mathrm{~m}$. 
distance and weakly affects the results of a test with a large inter-well distance.

\section{Conclusions}

This study develops a two-dimensional nonaxisymmetrical mathematical model to investigate the effect of finite mixing volume in the injection well bore on solute transport in a convergent radial tracer test. The LTFD method is applied to solve the advection-dispersion equation in cylindrical coordinates. The accuracy of the present model is verified using the Moench's axisymmetrical analytical solution. Comparing the analytical solution reveals that, by using an extra fine grid mesh and the upstream weighting technique, the LTFD model can effectively and accurately solve the two-dimensional radial advection-dispersion equation with large Peclet numbers. The LTFD model yields both nonaxisymmetrical concentration contours and breakthrough curves at the extraction well and the observation point. The concentration contours obtained through simulation show that the injection well bore mixing slows the migration of the tracer plume from the injection well to the extraction well. The results also show that the effect of the injection well bore mixing volume decreases as the inter-well distance increases.

\section{Acknowledgements}

The authors would like to thank the National Science Council of the Republic of China for financially supporting this work under Contract No. NSC 90-2313-B-242-004. We would also like to thank Professor Logan, who brought our attention to the problem of well bore mixing volume, and two anonymous reviewers who provided valuable suggestions and many key refinements.

\section{Appendix A}

The LTFD solution of the governing Eq. (12), subject to initial and boundary conditions (13) $-(20)$ is derived.
First, taking the Laplace transform of Eqs. (12), (13) and the associated boundary conditions (13)(20), with respect to $t_{\mathrm{D}}$ yields,

$$
\begin{aligned}
& \frac{1}{P e} \frac{1}{r_{\mathrm{D}}} \frac{\partial^{2} G}{\partial r_{\mathrm{D}}^{2}}+\frac{1}{r_{\mathrm{D}}} \frac{\partial G}{\partial r_{\mathrm{D}}}+\frac{1}{P e} \frac{a_{\mathrm{D}}}{r_{\mathrm{D}}^{3}} \frac{\partial^{2} G}{\partial \theta^{2}} \\
& =\frac{2 R}{1-r_{\mathrm{WD}}^{2}} s G \\
& \mu_{\mathrm{W}} s G=\frac{1}{P e} \frac{\partial G\left(r_{\mathrm{D}}, \theta, s\right)}{\partial r_{\mathrm{D}}} \quad \text { at } r=r_{\mathrm{W}} \\
& \frac{1}{P e} \frac{\partial G\left(r_{\mathrm{D}}, \theta, s\right)}{\partial r_{\mathrm{D}}}+G\left(r_{\mathrm{D}}, \theta, s\right) \\
& =\left\{\begin{array}{l}
G_{\mathrm{I}}(s) \\
0 \\
0
\end{array}-\delta<\theta<\pi\right. \\
& -G_{\mathrm{I}}(s)=\mu_{\mathrm{I}}\left[s G_{\mathrm{I}}(s)-C_{0}\right] \\
& \frac{\partial G\left(r_{\mathrm{D}}, 0, s\right)}{\partial \theta}=0 \\
& \frac{\partial G\left(r_{\mathrm{D}}, \pi, s\right)}{\partial \theta}=0
\end{aligned}
$$

where $s$ denotes the Laplace transform parameter and $G$ represents the Laplace transform of $C$, as defined by,

$$
\begin{aligned}
& G\left(r_{\mathrm{D}}, \theta, s\right)=\int_{0}^{\infty} C\left(r_{\mathrm{D}}, \theta, t_{\mathrm{D}}\right) \mathrm{e}^{-s t_{\mathrm{D}}} \mathrm{d} t_{\mathrm{D}} \\
& G_{\mathrm{I}}(s)=\int_{0}^{\infty} C_{\mathrm{I}}\left(t_{\mathrm{D}}\right) \mathrm{e}^{-s t_{\mathrm{D}}} \mathrm{d} t_{\mathrm{D}}
\end{aligned}
$$

Taking the finite Fourier cosine transform with respect to $\theta$ of Eqs. (A1)-(A6) yields,

$$
\begin{aligned}
& \frac{1}{P e} \frac{1}{r_{\mathrm{D}}} \frac{\mathrm{d}^{2} W}{\mathrm{~d} r_{\mathrm{D}}^{2}}+\frac{1}{r_{\mathrm{D}}} \frac{\mathrm{d} W}{\mathrm{~d} r_{\mathrm{D}}} \\
& -\left[\frac{1}{P e} \frac{a_{\mathrm{D}} n^{2}}{r_{\mathrm{D}}^{3}}+\frac{2 R s}{1-r_{\mathrm{WD}}^{2}}\right] W=0 \\
& \mu_{\mathrm{W}} s W=\frac{1}{P e} \frac{\partial W\left(r_{\mathrm{D}}, n, s\right)}{\partial r_{\mathrm{D}}} \text { at } r=r_{\mathrm{W}} \\
& \frac{1}{P e} \frac{\partial W\left(r_{\mathrm{D}}, n, s\right)}{\partial r_{\mathrm{D}}}+W\left(r_{\mathrm{D}}, n, s\right)=F(n)
\end{aligned}
$$


where

$$
\begin{aligned}
& F(n)= \begin{cases}G_{\mathrm{I}}(s) \delta, & n=0 \\
G_{\mathrm{I}}(s)\left[\frac{(-1)^{n} \sin n \delta}{n}\right], & n=1,2,3 \ldots\end{cases} \\
& G_{\mathrm{I}}(s)=\frac{\mu_{\mathrm{I}} C_{0}}{1+\mu_{\mathrm{I}} s}
\end{aligned}
$$

where $n$ denotes the finite Fourier cosine transform parameter and $W$ represents the finite Fourier cosine transform of $G$, as defined by,

$W\left(r_{\mathrm{D}}, n, s\right)=\int_{0}^{\pi} G\left(r_{\mathrm{D}}, \theta, s\right) \cos (n \theta) \mathrm{d} \theta$

Such a transform is useful since the inversion is directly given by the following formula (Sneddon, 1972)

$$
\begin{aligned}
G\left(r_{\mathrm{D}}, \theta, s\right)= & \frac{1}{\pi} W\left(r_{\mathrm{D}}, 0, s\right) \\
& +\frac{2}{\pi} \sum_{n=1}^{\infty} W\left(r_{\mathrm{D}}, n, s\right) \cos (n \theta)
\end{aligned}
$$

A finite difference method is applied by discretizing the radial distance of the transformed ordinary differential equation (A9). The advection terms are approximated by the upwind difference formulae. Substituting the difference formulae into the transformed partial differential equation yields the following algebraic equation

$$
\begin{gathered}
\frac{1}{P e} \frac{1}{\left(r_{\mathrm{D}}\right)_{i}} \frac{W_{i+1}-2 W_{i}+W_{i-1}}{\Delta\left(r_{\mathrm{D}}\right)^{2}}+\frac{1}{\left(r_{\mathrm{D}}\right)_{i}} \frac{W_{i+1}-W_{i}}{\Delta\left(r_{\mathrm{D}}\right)} \\
-\left[\frac{1}{P e} \frac{a_{\mathrm{D}} n^{2}}{\left(r_{\mathrm{D}}\right)_{i}^{3}}+\frac{2 R s}{1-r_{\mathrm{WD}}^{2}}\right] W_{i}=0
\end{gathered}
$$

The finite-difference equation in the Laplace domain is rearranged:

$$
a W_{i+1}+b W_{i}+c W_{i-1}=d
$$

where,

$$
\begin{aligned}
a= & \frac{1}{P e\left(r_{\mathrm{D}}\right)_{i} \Delta\left(r_{\mathrm{D}}\right)^{2}}+\frac{1}{\left(r_{\mathrm{D}}\right)_{i} \Delta\left(r_{\mathrm{D}}\right)}, \\
b= & -\left[\frac{2}{P e\left(r_{\mathrm{D}}\right)_{i} \Delta\left(r_{\mathrm{D}}\right)^{2}}+\frac{1}{\left(r_{\mathrm{D}}\right)_{i} \Delta\left(r_{\mathrm{D}}\right)}\right. \\
& \left.+\frac{a_{\mathrm{D}} n^{2}}{P e\left(r_{\mathrm{D}}\right)_{i}^{3}}+\frac{2 R s}{1-r_{\mathrm{WD}}^{2}}\right], \\
c= & \frac{1}{P e\left(r_{\mathrm{D}}\right)_{i} \Delta\left(r_{\mathrm{D}}\right)^{2}} \quad \text { and } d=0 .
\end{aligned}
$$

Written in matrix notation, the finite difference system of simultaneous equations (A15) becomes,

$[\Omega][W]=[D]$

where $[\Omega]$ denotes the coefficient matrix; $[W]$ represents the vector of the unknown transformed concentration, and $[D]$ is the known right-hand vector. The system of algebraic equations represented by Eq. (A16), can be solved using direct Gaussian elimination or other (for example, iterative) methods to yield a Laplace-transformed concentration at the node points. Additionally, a FORTRAN subroutine, DLSACB, is readily available (see Visual Numerics, Inc., 1994). The solutions in the original domain, $C\left(r_{\mathrm{D}}, \theta, t_{\mathrm{D}}\right)$, are the Laplace and finite Fourier cosine inversions of $W\left(r_{\mathrm{D}}, n, s\right)$. For convenience, the finite Fourier cosine transform is performed first. Also, the Laplace inverse of $W\left(r_{\mathrm{D}}, n, s\right)$ must be determined numerically. A FORTRAN subroutine DINLAP/ INLAP (Visual Numerics, Inc., 1994), based on the De Hoog et al. (1982) algorithm, is employed to perform the Laplace inversion. The computer code of the LTFD model is available from the corresponding author upon request.

\section{References}

Becker, M.W., Charbeneau, R.J., 2000. First-passage-time transfer functions for groundwater tracer tests conducted in radially convergent flow. J. Contam. Hydrol. 40, 299-310.

Chen, J.S., Liu, C.W., Chen, C.S., Yeh, H.D., 1996. A Laplace transform solution for tracer test in a radially convergent flow with upstream dispersion. J. Hydrol. 183, $263-275$

Chen, J.S., Chen, C.S., Gau, H.S., Liu, C.W., 1999. A two-well method to evaluate transverse dispersivity for tracer tests in a radially convergent flow field. J. Hydrol. 223, 175-197. 
De Hoog, F.R., Knight, J.H., Stokes, A.N., 1982. An improved method of Laplace transforms using a Fourier series approximation. SIAM J. Sci. Stat. Comput. 3 (3), 357-366.

Drost, W., Klotz, D., Koch, A., Moser, H., Neumaier, F., Rauert, W., 1968. Point dilution methods of investigating ground water flow by means of radioisotopes. Water Resour. Res. 4 (1), 125-146.

Guvanasen, V., Guvanasen, V.M., 1987. An approximate semianalytical solution for tracer injection tests in a confined aquifer with a radially convergent flow field and finite volume of tracer and chase fluid, Water Resour. Res. 23 (8), 1607-1619.

Hodgkinson, D.P., Lever, D.A., 1983. Interpretation of a field experiment on transport of sorbed and non-sorbed tracers through a fracture in crystalline rock. Radioact. Waste. Manage. Nucl. Fuel Cycle 4 (2), 129-158.

Mackay, D.M., Bianchi-Mosquera, G., Kopania, A.W., Kianjah, H., 1994. A forced-gradient experiment on solute transport in the Borden aquifer, 1; experimental methods and moment analysis of results. Water Resour. Res. 30 (2), 369-383.

Moench, A.F., 1989. Convergent radial dispersion: a Laplace transform solution for aquifer tracer testing. Water Resour. Res. 25 (3), 439-447.

Moench, A.F., 1991. Convergent radial dispersion: a note on evaluation of the Laplace transform solution. Water Resour. Res. 27 (12), 3261-3264.
Moench, A.F., 1995. Convergent radial dispersion in a doubleporosity aquifer with fracture skin: analytical solution and application to a field experiment in fractured chalk. Water Resour. Res. 31 (8), 1823-1835.

Sauty, J.-P., 1980. An analysis of hydrodispersive transfer in aquifers. Water Resour. Res. 16 (1), 145-158.

Sneddon, I.N., 1972. The Use of Integral Transform, McGraw-Hill, New York.

Thorbjarnson, K., Mackay, D.M., 1994. A forced gradient experiment on solute transport in the Borden aquifer, 2; transport and dispersion of the conservative tracer. Water Resour. Res. 30 (2), 385-399.

Visual Numerics, Inc., 1994. IMSL User's Manual. Houston, TX, vol. 1, pp. 159-161

Wang, H.Q., Crampon, N., 1995. Method for interpreting tracer experiments in radial flow using modified analytical solutions. J. Hydrol. 165, 11-31.

Welty, C., Gelhar, L.W., 1994. Evaluation of longitudinal dispersivity from nonuniform flow tracer tests. J. Hydrol. 153, 71-102.

Zlotnik, V.A., Logan, J.D., 1996. Boundary conditions for convergent radial tracer tests and the effect of well bore mixing volume. Water Resour. Res. 32 (7), 2323-2328. 